\title{
An attitudinal DICE distance based IVHF-TODIM for selecting agricultural sensors under IOT environment
}

\author{
Yang Zhao \\ Institute of Quality Development, Kunming University of \\ Science and Technology Kunming 650093, China \\ 749957683@qq.com
}

\author{
Tie-Dan Wang, Ding-Hong Peng \\ Institute of Quality Development, Kunming University of \\ Science and Technology Kunming 650093, China \\ pengdinghong2006@163.com
}

\begin{abstract}
The sensor is the foundation of agricultural IOT Technology, which is responsible for the networking information collection. So it is important for the enterprise to choose the suitable agricultural sensor, in order to solve this problem, this paper uses TODIM method for the sensor evaluation. In order to fully consider the expert opinion, the interval-valued hesitant fuzzy set is used, in order to avoid information loss DICE distance is proposed in the calculation of dominance degree. Finally, the improved IVHFTODIM is applied to the sensor.
\end{abstract}

Keywords-agricultural IOT Technology; sensor; Intervalvalued hesitant fuzzy set; TODIM method; DICE distance

\section{INTRODUCTION}

Agricultural IOT (Internet of things)[1] refers to the use of the sensor nodes to collect the real-time information of crop production environment, the monitoring system is composed of network technology, helping farmers to find problems, and accurately determine the location of the problem. In this way, agriculture will gradually shift from labor centered to information and software intelligent production model, so as to achieve the purpose of efficient agricultural production. While sensor is the foundation of agricultural IOT Technology, it can quantify the changes of the various things in the real world. At present, there are many kinds of sensors used in agriculture from the grow seeding, growth harvest until storage, the sensor technology has been widely used. Therefore, it is extremely important to select suitable sensor in the environment of agricultural IOT. Many of the attributes should be considered when choose an agricultural sensor including stability reliability applicability and safety. In view of selecting the suitable sensor, experts are based on the actual situation combing with their own knowledge as well as their psychological behavior to evaluate. This paper uses TODIM method (an acronym in Portuguese of interactive and multiple attribute decision making) to evaluate the sensor.

The TODIM[2] method, first proposed by Gomes and Lima in 1991, it is a discrete multi-criteria method based on prospect theory[3] and has been proven to be a valuable tool for solving the MCDM problem considering the DM's behavior. The TODIM method is based on the description provided by people's experience, it is designed to help people to effectively make decisions in the face of risk, and can adjust the

*Corresponding author. E-mail addresses: pengdinghong2006@163.com (D.-H. Peng). parameters in the process of calculation according to the risk preferences of decision makers.

However, with the increasing complexity of social and economic problems and the limited understanding of things, it is becoming more and more difficult for people to express their uncertain judgment about reality by precise numbers. In order to solve the ambiguity in the decision-making process, Zadeh[4](1965) proposed the fuzzy sets to deal with imprecise and vague information, it has been widely used in various fields. This theory has attracted the attention of many scholars, subsequently, many foreign scholars extended the fuzzy sets, proposing the intuitionistic fuzzy sets type-2 fuzzy sets and hesitant fuzzy sets[5], but the scholars find that in real life, due to insufficiency in available information, it may be difficult for the decision makers to exactly express their opinions with some crisp values or the others, but we can use some interval values within $[0,1]$, it is easier and more reasonable, based on the continuous study of fuzzy sets, $\mathrm{Xu}[6]$ proposed the concept of interval-valued hesitant fuzzy sets on the basis of fuzzy sets which permit the membership degree of an element to a given set with several different interval values, adding flexibility to the decision maker's assignment, it can describe the uncertainty of the decision-maker more precisely, and is especially suitable for describing the realistic decision problem with hesitation. So we can use the IVHF sets to describe the attribute of the sensor.

Summarizing the aforementioned discussions, this paper proposes improved TODIM method for agricultural IOT sensor choosing, it can take the DMs' attitudinal character into account. And in order to adapt to the development requirements of agricultural scale, precision and application, this paper select stability, reliability, and security as the indicators to ensure that the sensor can be used in the open and harsh environment.

\section{Preliminaries}

A. Sensor

The sensor is a device that can feel the measured information and convert it to the usable signal according to the certain rule. The function of the sensor is like the human senses, to feel the change of the environment from different aspects. . Along with the agricultural equipment to automation, information and intelligent direction, various kinds of sensors have been widely used in agricultural equipment, realizing the requirements of accuracy precision and high efficiency. Moreover sensor is the bottom of the whole system, it is one of the basic source of all collected data, This determines the 
sensor application in agriculture are exposed to the environment is bad, often in outdoor withstand wind, sun, rain and other weather test and on the performance of agricultural sensor requirements are more strict, such as whether to keep the sensor performance is good for a long time, its stability is good, the life is long enough, the measuring range is constant, and so on, these are all factors to consider agricultural sensors[7]

\section{B. Interval-valued Hesitant fuzzy sets}

Interval-valued hesitant fuzzy set (IVHFS), is a powerful tool to account for inherent hesitancy of human judgments in daily life. It has been widely used for many fields.

Definition 1[6]: Let $\mathrm{X}$ be a reference set, and it is the set of all closed subintervals of $[0,1]$ which can be represented as the following mathematical symbol:

$$
\begin{gathered}
\tilde{E}=\left\{<x, \tilde{h}_{E}(x)>\mid x \in X\right\} \\
\tilde{h}_{\tilde{E}}(x)=\left\{\tilde{\gamma}=\left[\tilde{\gamma}^{L}, \tilde{\gamma}^{U}\right]\right\}
\end{gathered}
$$

Where $\tilde{h}_{E}(x)$ is a set of values in $[0,1]$, denoting the all possible interval-valued membership values of the element $x \in X$ to the set $\tilde{E}$.

Definition 2[8]: For a IVHFE

$\tilde{h}=<\left[\tilde{\gamma}_{1}^{L}, \tilde{\gamma}_{2}^{U}\right],\left[\tilde{\gamma}_{2}^{L}, \tilde{\gamma}_{2}^{U}\right], \ldots\left[\tilde{\gamma}_{l(\tilde{h})}^{L}, \tilde{\gamma}_{l(\tilde{h})}^{U}\right]>$ we can get a new measured function $Z_{\delta}(h)$ to measure the two IVHFEs degree, it can be defined as follows:

$Z_{\delta}(\tilde{h})=\left(\sum_{j=1}^{l(\tilde{h})}\left(\tilde{\gamma}_{j}^{L}\right)^{\delta} / l(\tilde{h})\right)^{\frac{1}{\delta}}+\left(\sum_{j=1}^{l(\tilde{h})}\left(\tilde{\gamma}_{j}^{U}\right)^{\delta} / l(\tilde{h})\right)^{\frac{1}{\delta}}$

Where $l(h)$ is the number of the elements in $h$, and the $\delta(0<\delta \leq 1)$ is a parameter determined by the DM which is variable, it can be tuned according to the decision attitude.

\section{Dice distance for IVHFS}

Distance measure is the vital concept for the calculation of dominance degree, but many distances suffer from defects of the IVHFSs, in order to more accurately calculate their distances, considering that the numbers of values in different IVHFEs, they need to subjectively add (or remove) some interval values to the IVHFE with fewer (or more) value, thus it will make information loss and distortion. While Dice distance measure is through the set operation to describe the degree of similarity between two vectors, it does not need to subjectively add (or remove) some interval values to the IVHFE with fewer (or more) values, thus it can efficiently avoid information loss and distortion. So in this paper, we will use the DICE distance in the dominance degree. COWA operator is a powerful tool for Information fusion. And there we use the HFCOWA operator can retain more information of original interval-type information and taking into account of the DMs' attitudinal character.
Definition 3[9]: A HFCOWA operator is a mapping $\tilde{\delta}_{Q}$ :

$\tilde{h} \rightarrow h$, which has associated with it a BUM function,

$Q:[0,1] \rightarrow[0,1]$, and it has three properties

(1) $Q(0)=0$ (2) $Q(1)=1$ (3) $Q(x) \geq Q(y) x>y$,

$$
\begin{aligned}
& \tilde{\delta}_{Q}(\tilde{h})=\bigcup_{\tilde{\gamma} \in \tilde{h}}\left\{f_{Q}(\tilde{\gamma})\right\}= \\
& \bigcup_{\tilde{\gamma} \in \tilde{h}}\left\{\int_{0}^{1} \frac{d Q(y)}{d y}\left[\tilde{\gamma}^{U}-\left(\tilde{\gamma}^{U}-\tilde{\gamma}^{L}\right) y\right] d y\right\}
\end{aligned}
$$

There, $\lambda=\int_{0}^{1} Q(y) d y$ is the attitudinal character of $\mathrm{Q}$, we can use the attitudinal character to simply the above Eqs (2), the $\tilde{\delta}_{Q}$ can be simplified as follows:

$\tilde{\delta}_{Q}(\tilde{h})=\bigcup_{\tilde{\gamma} \in \tilde{h}}\left\{(1-\lambda) \tilde{\gamma}^{L}+\lambda \tilde{\gamma}^{U}\right\}$

It is obvious that the we can use the HFCOWA operator to transform the IVHFE into a HFE by a attitudinal character parameter $\lambda$.

$D$ Improved Dice distance measure

We can use the Dice distance measure to measure the degree of similarity between two sets, but the original Dice similarity measure induce this undefined situation when one vector is zero, so in this paper we put forward the improved Dice distance measure to overcome the defects.

Definition4: Let $X=\left(x_{1}, x_{2}, \ldots, x_{n}\right)$ and $Y=\left(y_{1}, y_{2}, \ldots y_{n}\right)$, be the two vectors of length $n$ where all the coordinates are positive. Then the Dice distance measure is defined as follows: $d^{\text {Dice }}(X, Y)=2\left(1-\frac{2 \min \left(X_{i}, Y_{i}\right)+1}{X_{i}+Y_{i}+1}\right)$

The Dice similarity measure satisfies the following properties:

(1) $0 \leq D(X, Y) \leq 1$;

(2) $D(X, Y)=D(Y, X)$

(3) $D(X, Y)=0$ if and only if $\mathrm{X}=\mathrm{Y}$

From the equation, we can know that when the vector is zero the equation is meaningful.

For example: Let $\mathrm{X}=0, \mathrm{Y}=0$, then the DICE distance measure between 0 as follows:

$$
d^{\text {Dice }}(0,0)=2\left(1-\frac{2 \min (0,0)+1}{0+0+1}\right)=0
$$

III. Attitudinal Dice distance measures for IVHFSs

Dice distance measure is used to measure the difference between the interval-valued hesitant fuzzy sets, it does not need to subjectively add (or remove) some interval values to the IVHFE with fewer (or more) values, and then when we use the HFCOWA operator it can retain more information of original interval type information, but also allow their computation by taking into account of the DMs' attitudinal character. So the IVHF-DICE distance is very suitable for the dominance degree. 
Definition5:

Let $\tilde{h}_{a}=\bigcup_{\tilde{\gamma}_{a} \in \tilde{h}_{a}}\left\{\tilde{\gamma}_{a}=\left[\tilde{\gamma}_{a}^{L}, \tilde{\gamma}_{a}^{U}\right]\right\}, \tilde{h}_{b}=\bigcup_{\tilde{\gamma}_{b} \in \tilde{h}_{b}}\left\{\tilde{\gamma}_{b}=\left[\tilde{\gamma}_{b}^{L}, \tilde{\gamma}_{b}^{U}\right]\right\}$ be two IVHFEs, then

$$
\begin{aligned}
& d_{Q}\left(\tilde{h}_{a}, \tilde{h}_{b}\right)=d\left(\tilde{\delta}_{Q}\left(\tilde{h}_{a}\right), \tilde{\delta}_{Q}\left(\tilde{h}_{b}\right)\right) \\
& =\frac{1}{\# \tilde{h}_{a} \# \tilde{h}_{b}} \sum_{\tilde{\gamma}_{a} \in \tilde{h}_{a}} \sum_{\tilde{\gamma}_{b} \in \tilde{h}_{b}} 2\left(1-\frac{2 \min \left(f_{Q}\left(\tilde{\gamma}_{a}\right), f_{Q}\left(\tilde{\gamma}_{b}\right)\right)+1}{f_{Q}\left(\tilde{\gamma}_{a}\right)+f_{Q}\left(\tilde{\gamma}_{b}\right)}\right)
\end{aligned}
$$

Then we can use the HFCOWA, the equation are given as follows:

$$
\begin{aligned}
& d_{Q}\left(\tilde{h}_{a}, \tilde{h}_{b}\right)=\frac{1}{\# \tilde{h}_{a} \# \tilde{h}_{b}} \times \sum_{\tilde{\gamma}_{a} \in \tilde{h}_{a}} \sum_{\tilde{\gamma}_{b} \in \tilde{h}_{b}} \\
& \left\{2\left\{\frac{2 \min \left(\int_{0}^{1} \frac{d Q(y)}{d y}\left[\tilde{\gamma}_{a}^{U}-\left(\tilde{\gamma}_{a}^{U}-\tilde{\gamma}_{a}^{L}\right) y\right] d y, \int_{0}^{1} \frac{d Q(y)}{d y}\left[\tilde{\gamma}_{b}^{U}-\left(\tilde{\gamma}_{b}^{U}-\tilde{\gamma}_{b}^{L}\right) y\right] d y\right)+1}{\left(\int_{0}^{1} \frac{d Q(y)}{d y}\left[\tilde{\gamma}_{a}^{U}-\left(\tilde{\gamma}_{a}^{U}-\tilde{\gamma}_{a}^{L}\right) y\right] d y+\int_{0}^{1} \frac{d Q(y)}{d y}\left[\tilde{\gamma}_{b}^{U}-\left(\tilde{\gamma}_{b}^{U}-\tilde{\gamma}_{b}^{L}\right) y\right] d y\right)+1}\right)\right\}
\end{aligned}
$$

If $\lambda=\int_{0}^{1} Q(y) d y$ is the attitudinal character of $\mathrm{Q}$, then the operation of can be simplified as follows:

$$
\begin{aligned}
& d_{Q}\left(\tilde{h}_{a}, \tilde{h}_{b}\right)=\frac{1}{\# \tilde{h}_{a} \# \tilde{h}_{b}} \times \sum_{\tilde{\gamma}_{a} \in \tilde{h}_{a}} \sum_{\tilde{\gamma}_{b} \in \tilde{h}_{b}} \\
& 2 \times\left(1-\frac{2 \min \left\{\left[(1-\lambda) \tilde{\gamma}_{a}^{L}+\lambda \tilde{\gamma}_{a}^{U}\right],\left[(1-\lambda) \tilde{\gamma}_{b}^{L}+\lambda \tilde{\gamma}_{b}^{U}\right]\right\}+1}{(1-\lambda) \tilde{\gamma}_{a}^{L}+\lambda \tilde{\gamma}_{a}^{U}+(1-\lambda) \tilde{\gamma}_{b}^{L}+\lambda \tilde{\gamma}_{b}^{U}+1}\right)
\end{aligned}
$$

\section{Description of the improved IVHF-TODIM method}

Based on the above studies, this paper propose the improved IVHF-TODIM method, this method can fully reflects the DM's behavioral characteristic such as reference dependence and loss aversion, and the HFCOWA operator can get more information and also make the decision-making results more accurate.

For the sake of simplicity, let that $A=\left\{A_{1}, A_{2}, \ldots A m\right\}$ is a set of alternative programs, $C=\left\{C_{1}, C_{2}, \ldots C_{n}\right\}$ is a set of criteria, $w=\{w 1, w 2, \ldots w n\}$ is a set of weight, $\sum_{j=1}^{n} w_{j}=1$ $w_{j} \in[0,1]$.

Step 1: Identify the decision matrix $A=\left[a_{i j}\right]_{m \times n}$ and then standardize decision matrix $A=\left[a_{i j}\right]_{m \times n}$ into $B=\left[b_{i j}\right]_{m \times n}$

Step 2: Calculate the relative weight $w_{j}{ }^{\prime}$ of the criterion $C_{j}$ to the reference criterion $C^{*}$ according to the following expression:

$w_{j}^{\prime}=w_{j} / w^{*}, j \in M$

Where $w_{j}$ is the weight value of the criterion $C_{j}$ and $w^{*}=\max \{w 1, w 2 \ldots . . . w n\}$ is the reference weight.

Step 3: Calculate the Dice distance of each alternative $A_{i}$ over each alternative $A_{k}$ according to the criteria $C_{j}$ using the following expression:

$$
\begin{aligned}
& d_{\lambda}\left(A_{i}, A_{k}\right)=\frac{1}{\# \tilde{h}_{i} \# \tilde{h}_{k}} \sum_{\tilde{\gamma}_{i} \in \tilde{h}_{i}} \sum_{\tilde{\gamma}_{k} \in \tilde{h}_{k}} \\
& 2 \times\left(1-\frac{2 \min \left\{\left[(1-\lambda) \tilde{\gamma}_{i}^{L}+\lambda \tilde{\gamma}_{i}^{U}\right],\left[(1-\lambda) \tilde{\gamma}_{k}^{L}+\lambda \tilde{\gamma}_{k}^{U}\right]\right\}+1}{(1-\lambda) \tilde{\gamma}_{i}^{L}+\lambda \tilde{\gamma}_{i}^{U}+(1-\lambda) \tilde{\gamma}_{k}^{L}+\lambda \tilde{\gamma}_{k}^{U}+1}\right)
\end{aligned}
$$

Step 4: Calculate the dominance of each alternative $A_{i}$ over each alternative $A_{k}$ using the following expression:

When the $Z_{\varsigma}\left(\tilde{h}_{i j}\right)-Z_{\varsigma}\left(\tilde{h}_{k j}\right)>0$

$$
\delta\left(A_{i}, A_{k}\right)==\sqrt{\left\{d^{\text {Dice }}\left(\tilde{h}_{i j}, \tilde{h}_{k j}\right)\right\} \times w_{j k} / \sum_{j=1}^{m} w_{j k}},
$$

When the $Z_{\varsigma}\left(\tilde{h}_{i j}\right)-Z_{\varsigma}\left(\tilde{h}_{k j}\right)<0$

$$
\delta\left(A_{i}, A_{k}\right)=-1 / \theta \times \sqrt{d^{\text {Dice }}\left(\tilde{h}_{i j}, \tilde{h}_{k j}\right) \times \sum_{j=1}^{m} w_{j k} / w_{j k}}
$$

When the $Z \varsigma\left(\tilde{h}_{i j}\right)-Z \varsigma\left(\tilde{h}_{k j}\right)=0$

$$
\delta\left(A_{i}, A_{k}\right)=0
$$

Where the parameter $\theta$ represents the attenuation factor of the losses, when $\theta>1$, the decision makers are risk averse, when $\theta<1$, the decision maker's is risk preference.

Step 5: Calculate the overall prospect value of the alternative $A_{i}$ according to the following expression:

Firstly, from the individual dominance degree, we can get the overall dominance of the alternative $A_{i}$ over the alternative $A_{k}$.

$$
\varphi\left(A_{i}, A_{k}\right)=\sum_{l=1}^{n} \delta\left(A_{i}, A_{k}\right),(i, k \in N)
$$

Then calculate the overall dominance degree of $A_{i}$ over others, using the following expression:

$$
T\left(A_{i}\right)=\sum_{k=1}^{m} \varphi\left(A_{i}, A_{k}\right)
$$

Step 6: Normalize the overall dominance degree:

$$
S\left(A_{i}\right)=\frac{T\left(A_{i}\right)-\min \left\{T\left(A_{i}\right)\right\}}{\max \left\{T\left(A_{i}\right)\right\}-\min \left\{T\left(A_{i}\right)\right\}}, i \in N
$$

According to the $S\left(A_{i}\right)$, the greater the value, the better the alternative.

With the development of wireless communication technology, especially the research and development of Internet of things technology and its application platform, through the application of Internet of things technology to provide important support and platform for modern agricultural development. It has become one of the focuses of modern industry, agriculture and academia, while sensor is the foundation of agricultural IOT Technology, it can quantify the changes of the various things in the real world. 
In order to improve the modern production of agriculture, an enterprise of Henan wants to choose the suitable sensor, it construct a panel of experts to evaluate the three sensors $A_{1} A_{2} A_{3}$ according to the four attributes stability $C_{1}$ reliability $C_{2}$ applicability $C_{3}$ and safety $C_{4}$, the experts found that the reliability is considered to the most important factor, therefore the weight vector of the criteria is $w=\{0.2,0.35,0.15,0.25\}$. Supposing that the experts give the rating values by using IVHFEs, and standardizing it according to the value is benefit, the decision matrix is constant and then the interval-valued hesitant fuzzy decision matrix is presented in table 1 .

\begin{tabular}{ccccc}
\multicolumn{5}{c}{ Tab1 The evaluation of the estimate } \\
\hline & $\mathrm{C}_{1}$ & $\mathrm{C}_{2}$ & $\mathrm{C}_{3}$ & $\mathrm{C}_{4}$ \\
\hline $\mathrm{A}_{1}$ & $\{[0.5,0.6],[$ & $\{0.5,0.8\}$ & $\{[0.4,0.5],[$ & $\{0.4,0.7\}$ \\
& $0.8,0.9]\}$ & & $0.6,0.8]\}$ & \\
$\mathrm{A}_{2}$ & {$[0.4,0.5],[0$.} & $\{0.5,0.7\}$ & $\{0.6,0.8\}$ & {$[0.3,0.4],[0$.} \\
& $6,0.7]$ & & & $5,0.8]$ \\
$\mathrm{A}_{3}$ & $\{0.4,0.55\}$ & $\{[0.2,0.4],[$ & $\{0.4,0.5\}$ & $\{0.4,0.6\}$ \\
& & $0.6,0.7]\}$ & & \\
\hline
\end{tabular}

Firstly, calculate the relative weight, the weight vector of the criteria is $w=\{0.2,0.35,0.15,0.25\}$, using the equation (6), we can get the relative weight vector of the criteria is $w=\{0.57,1,0.43,0.57\}$, and secondly using the equation(4) to calculate the Dice distance of each alternative $A_{i}$ over each alternative $A_{k}$, listed in table 2-5.

Tab 2 The distance of alternative over others according to the $C_{1}$

\begin{tabular}{|c|c|c|c|}
\hline & $\mathrm{A}_{1}$ & $\mathrm{~A}_{2}$ & $\mathrm{~A}_{3}$ \\
\hline$\overline{\mathrm{A}_{1}}$ & 0 & 0.1 & 0.36 \\
\hline $\mathrm{A}_{2}$ & 0.1 & 0 & 0.83 \\
\hline $\mathrm{A}_{3}$ & 0.36 & 0.83 & 0 \\
\hline \multicolumn{4}{|c|}{ Tab 3 The distance of alternative over others according to the $\mathrm{C}_{2}$} \\
\hline & $\mathrm{A}_{1}$ & $\mathrm{~A}_{2}$ & $\mathrm{~A}_{3}$ \\
\hline $\mathrm{A}_{1}$ & 0 & 0.03 & 0.42 \\
\hline $\mathrm{A}_{2}$ & 0.03 & 0 & 0.42 \\
\hline $\mathrm{A}_{3}$ & 0.42 & 0.42 & 0 \\
\hline \multicolumn{4}{|c|}{ Tab 4 The distance of alternative over others according to the $\mathrm{C}_{3}$} \\
\hline & $\mathrm{A}_{1}$ & $\mathrm{~A}_{2}$ & $\mathrm{~A}_{3}$ \\
\hline $\mathrm{A}_{1}$ & 0 & 0.26 & 0.61 \\
\hline $\mathrm{A}_{2}$ & 0.26 & 0 & 0.63 \\
\hline $\mathrm{A}_{3}$ & 0.61 & 0.63 & 0 \\
\hline \multicolumn{4}{|c|}{ Tab 5 The distance of alternative over others according to the $\mathrm{C}_{4}$} \\
\hline & $\mathrm{A}_{1}$ & $\mathrm{~A}_{2}$ & $\mathrm{~A}_{3}$ \\
\hline $\mathrm{A}_{1}$ & 0 & 0.32 & 0.07 \\
\hline $\mathrm{A}_{2}$ & 0.32 & 0 & 0.29 \\
\hline $\mathrm{A}_{3}$ & 0.07 & 0.29 & 0 \\
\hline
\end{tabular}

Then we can use the equation (1) to calculate the gains or lost of the alterative $A_{i}$ over the $A_{k}$, taking $\varsigma=0.1$, we can obtain the advantage-disadvantage between alternatives, listed in table 6. Meanwhile, we can take $\theta=1$, next the following values can be calculated:

(1) Aiming at the attributes $C_{1} C_{2} C_{3} C_{4}$, the dominance degree of $A_{1}$ over the $A_{2}$ and the dominance degree of $A_{1}$ over the $A_{3}$.

(2) Using the equation(7) to add the above values, we can get the overall dominance degree between $A_{1}$ and others, similarly, we can get the dominance degree of each alterative over others
Tab 6 advantage and disadvantage

\begin{tabular}{|c|c|c|c|}
\hline & $\mathrm{A}_{1} / \mathrm{A}_{2}$ & $\mathrm{~A}_{1} / \mathrm{A}_{3}$ & $\mathrm{~A}_{2} / \mathrm{A}_{3}$ \\
\hline $\mathrm{C}_{1}$ & advantage & advantage & advantage \\
\hline $\mathrm{C}_{2}$ & advantage & advantage & disadvantage \\
\hline $\mathrm{C}_{3}$ & advantage & advantage & advantage \\
\hline $\mathrm{C}_{4}$ & disadvantage & advantage & advantage \\
\hline \multicolumn{4}{|c|}{ Tab 7 The dominance degree of each alternative over the others. } \\
\hline & $\mathrm{A}_{1}$ & $\mathrm{~A}_{2}$ & $\mathrm{~A}_{3}$ \\
\hline $\mathrm{A}_{1}$ & 0 & -0.19 & -1.14 \\
\hline $\mathrm{A}_{2}$ & 0.19 & 0 & -0.37 \\
\hline $\mathrm{A}_{3}$ & 1.14 & 0.37 & 0 \\
\hline
\end{tabular}

Thirdly, using equation (8) and based on the above results in tables, we can get the overall dominance degree of alterative $A_{i}$ over all others, namely:

$$
T\left(A_{1}\right)=1.33 \quad T\left(A_{2}\right)=0.18 \quad T\left(A_{3}\right)=-1.51
$$

Finally, according to the above results, we use equation (9) to normalize the dominance degree, and the ranking order is determined.

$$
S\left(A_{1}\right)=1 \quad S\left(A_{2}\right)=0.6 \quad S\left(A_{3}\right)=0
$$

Apparently, $A_{1}$ is the most suitable sensor

\section{Summary and Conclusions}

This paper proposes an improved IVHF-TODIM method for the choose of the sensor. Agriculture sensor technology is the key of agricultural research and application of the Internet of things, the source of the information perception of things, so the choose of sensor is very important, we use the TODIM method for solving this problem, it is the very helpful tool, particularly considering the DM's attitude. Then the IVHF sets can retain the experts' decision-making information fully. Finally, we use the DICE distance measures in the dominance degree, it needn't subjectively add (or remove) some interval values to the IVHFE with fewer (or more) values, so the method applied to the choose of the sensor is useful.

Acknowledgment

This work is partially supported by the National Natural Science Foundation of China (61364016); China Postdoctoral Science Foundation(2015T80990,2014M550473); the Applied Basic Research Programs of Yunnan Province, China (2014FB136).

\section{References}

[1]UIT.ITU Internet Reports : The Internet of Things[R] .2005.

[2]Gomes L F A M, Lima M M P P. Todim: Basic and application to multicriteria ranking of projects with environmental impacts[J]. Foundations of Computing and Decision Sciences, 1992, 16(4):113-127.

[3]Kahneman D, Tversky A. Prospect theory: An analysis of decision under risk.[J]. Econometrica, 1990, 47(2):140-170.

[4]Zadeh L A. Fuzzy sets[J].Information and Control,1965,8:338-356.

[5]Torra V. Hesitant fuzzy sets[J].International Journal of Intelligent

Systems,2010,25:529-539.

[6]Chen N, Xu Z, Xia M. Interval-valued hesitant preference relations and their applications to group decision making[J]. Knowledge-Based Systems, 2013, 37(2):528-540.

[7]Doinea M, Boja C, Batagan L, et al. Internet of Things Based Systems for Food Safety Management[J]. Information Economica, 2015, 19(1/2015):87-97. [8]Zhang X, Xu Z. The TODIM analysis approach based on novel measured functions under hesitant fuzzy environment[J]. Knowledge-Based Systems, 2014, 61(2):48-58.

[9]Peng D H, Wang T D, Gao C Y, et al. Enhancing relative ratio method for MCDM via attitudinal distance measures of interval-valued hesitant fuzzy sets[J]. International Journal of Ma-chine Learning \& Cybernetics, 2016:12tion 